\section{Missing ethnic density data}

To investigate the concept of ethnic density one would have to draw a sample representative of the rich diversity of ethnicities in England. Modood \& Berthoud ${ }^{1}$ define ethnic group as:

'a community whose heritage offers important characteristics in common between its members and which makes them distinct from other communities. There is boundary, which separates "us" from "them", and the distinction would probably be recognised on both sides of that boundary. Ethnicity is a multi-faceted phenomenon based on physical appearance, subjective identification, cultural and religious affiliation, stereotyping, and social exclusion.

The study by Das-Munshi et $a l^{2}$ included White British, Irish, Black Caribbean, Bangladeshi, Indian and Pakistani respondents. In the EMPIRIC study, ethnicity was defined by self-assessment using the same categories as the 1991 Census. ${ }^{3}$ The 1991 Census collected data on nine ethnicity groups: White, Black Caribbean, Black African, Black other, Indian, Pakistani, Bangladeshi, Chinese and any other ethnic group. ${ }^{4}$ Das-Munshi et al's study has not looked at four of the nine ethnic subgroups listed and is therefore not representative of the ethnicities in England.

Das-Munshi et al have not explained the significant nonparticipation of candidates $(37.8 \%)$ in the study, making it difficult to draw conclusions from the data. Owing to various factors - including that of participants having moved out of the survey area or to an unknown new address, or the participant having died or reached the age of 75 after the sample was drawn $-11 \%$ of the sample was no longer eligible for interview. ${ }^{5}$

Of eligible individuals, refusals were received in person directly from the selected participant in $18 \%$ of cases, a further $2 \%$ refusing by post and $2 \%$ being proxy refusals. Most noncontacts resulted from the interviewer being unable to make contact with the participant, although there were also cases where no contact was made with anyone at the household after four or more telephone call attempts. The most common reason for any other unproductive outcome was that the participant was away on holiday or in hospital throughout the survey period. ${ }^{5}$

1 Modood T, Berthoud R. Ethnic Minorities in Britain: Diversity and Disadvantage - The Fourth National Survey of Ethnic Minorities. Policy Studies Institute, 1998

2 Das-Munshi J, Bécares L, Boydell JE, Dewey ME, Morgan C, Stansfeld SA, et al Ethnic density as a buffer for psychotic experiences: findings from a national survey (EMPIRIC). Br J Psychiatry 2012; 201: 282-90.

3 Weich S, Nazroo J, Sproston K, McManus S, Blanchard M, Erens B, et al. Common mental disorders and ethnicity in England: the EMPIRIC study. Psychol Med 2004; 34: 1543-51.

4 Office of Population Censuses and Surveys. 1991 Census: Definitions, Great Britain. HMSO, 1992

5 UK Data Archive. Ethnic Minority Psychiatric IIIness Rates In The Community (EMPIRIC): User Guide for UK Data Archive. UKDA, no date (http:// www.esds.ac.uk/doc/4685/mrdoc/pdf/4685userguide.pdf).

Hinesh Topiwala, CT2 in Psychiatry, Royal Edinburgh Hospital, UK. Email: Hinesh.Topiwala@nhslothian.scot.nhs.uk

doi: 10.1192/bjp.202.4.310

Authors' reply: Dr Topiwala raises two additional points which we have discussed in our paper. The original EMPIRIC survey omitted a number of ethnic minority groups. ${ }^{1}$ We were therefore unable to analyse associations for these groups as we did not have the data. As we mention in the 'Limitations' section of our paper, ${ }^{2}$ since this survey (2002) there have been a number of other new migrant groups to Britain, for example from Eastern Europe. Therefore we caution that the findings should not be generalised outside of the ethnic minority groups surveyed in our study.
The issue of non-response has been discussed in the Method under 'Survey design.'. Weights to account for non-response bias were derived using stepwise logistic regression techniques using data which were available from previous Health Surveys for England. ${ }^{1}$ A number of important demographic predictors of non-response were included in the models (including individual and household predictors as well as primary sampling units). ${ }^{1}$ Interactions by ethnicity were also included in the models, in order to account for the differing probabilities of response by ethnic group. ${ }^{1}$ As mentioned in our paper, wherever possible we used these survey weights to correct for non-response in our analyses. $^{2}$

1 Sproston J, Nazroo J. Ethnic Minority Psychiatric Illness Rates in the Community (EMPIRIC): Quantitative Report. TSO (The Stationery Office), 2002.

2 Das-Munshi J, Bécares L, Boydell JE, Dewey ME, Morgan C, Stansfeld SA, et al. Ethnic density as a buffer for psychotic experiences: findings from a national survey (EMPIRIC). Br J Psychiatry 2012; 201: 282-90.

Jayati Das-Munshi, Department of Health Service and Population Research, Section of Epidemiology, King's College London, Institute of Psychiatry, De Crespigny Park, London SE5 8AF, UK. Email: jayati.das-munshi@kcl.ac.uk

doi: 10.1192/bjp.202.4.310a

\section{Cardiovascular fitness and serious depression in adulthood}

The report by Åberg et $a l^{1}$ of an association between lower cardiovascular fitness at age 18 and serious depression in adulthood is interesting but their conclusion that the results 'strengthen the theory of a cardiovascular contribution to the aetiology of depression' is questionable. The authors recognise that although they controlled for psychiatric symptoms and disorders prior to and at the time of baseline assessment of cardiovascular fitness, they did not obtain information 'which could help in defining subsyndromal affective problems', nor details of 'other possible confounders such as personality, smoking and low self-esteem' that may increase risk for both poor fitness and depression. Seemingly lower cardiovascular fitness is not a risk factor for bipolar depression (or mania) and may not be a risk factor for females. ${ }^{2}$ It is not stated whether consideration was given to psychiatric disorders apart from depression (e.g. anxiety disorders) and this leaves uncertainty as to whether lower cardiovascular fitness at age 18 is only a risk factor for non-bipolar depression in males. Considering that all study participants were sufficiently fit for recruitment into national service, it would be difficult to explain how a mere difference in cardiovascular fitness could (as suggested by the authors) contribute to cause 'severe' depression even before the age of 30 . Given a recognised relationship between adverse developmental experiences, personality traits and depression, ${ }^{3,4}$ it would be important to obtain information about such potentially confounding variables as well as 'subsyndromal problems' at the time of assessing cardiovascular fitness. It is suggested that in light of such missing information, the reported finding does not as yet 'strengthen the theory of a cardiovascular contribution to the aetiology of depression'. It is more an interesting association that warrants further investigation.

1 Åberg MAl, Waern M, Nyberg J, Pedersen NL, Bergh Y, Åberg DN, et al. Cardiovascular fitness in males at age 18 and risk of serious depression in adulthood: Swedish prospective population-based study. Br J Psychiatry 2012; 201: 352-9.

2 Sund AM, Larsson B, Wichstrom L. Role of physical and sedentary activities in the development of depressive symptoms in early adolescence. Soc Psychiatry Psychiatr Epidemiol 2011; 46: 431-41. 